\title{
Diseño de un proceso de producción de ácido cítrico por simulación computacional.
}

\section{Design of a process of production of citrus acid by computational simulation}

María Augusta Guadalupe Alcoser. ${ }^{1}$, Mabel Mariela Parada Rivera. ${ }^{2}$, Linda Mariuxi Flores Fiallos. ${ }^{3} \&$ Paúl Marcelo Manobanda Pinto. ${ }^{4}$

Recibido: 18-10-2019 / Revisado: 15-11-2019 /Aceptado: 04-12-2019/ Publicado: 05-01-2020

\begin{abstract}
.
DOI: https://doi.org/10.32/cienciadigital.v3i1.947

The design of a citric acid production process from fermentation with Aspergillus Niger by computer simulation, was carried out with the use of SuperPro Designer software, the design parameters were established based on previous studies, which were entered into the calculation platform, subsequently the results obtained in the design were analyzed technically and economically, which allowed to determine an amount of citric acid production of 6914.69 ton / year, when using FOB prices (Free on board) you have an investment total of $\$ 31,460,000$ together with a NPV (net present value) of $\$$ 165,000 and an IRR (internal rate of return) of $8.05 \%$, indicating that the project can be carried out and is profitable in a time of retribution 8 years old The process consists of two stages: fermentation and isolation, which must be carried out under the operating conditions ( $\mathrm{T}=25^{\circ} \mathrm{C}$ and $\mathrm{P}=1$ atmosphere in most operations) and the optimal fermentation conditions for Aspergillus niger $\left(\mathrm{T}=35^{\circ} \mathrm{C}, \mathrm{pH}=5.8\right.$ and $\mathrm{P}=1$ atmosphere) that are suitable to obtain a quality product that can be offered in the national and international market. Finally, it is proven that the use of software allows technical studies to be developed without the need to resort to long periods of time with experimental laboratory tests, that is, it saves us: time, pre-design costs and errors.
\end{abstract}

Keywords: Chemical Engineering / Industrial Processes / Simulation/ Fermentation/ Operation parameters

\footnotetext{
${ }^{1}$ Escuela Superior Politécnica de Chimborazo, Chimborazo, Ecuador, maria.guadalupe@espoch.edu.ec

${ }^{2}$ Escuela Superior Politécnica de Chimborazo, Chimborazo, Ecuador, mparada@espoch.edu.ec

${ }^{3}$ Escuela Superior Politécnica de Chimborazo, Chimborazo, Ecuador, linda.flores@espoch.edu.ec

${ }^{4}$ Universidad Estatal Amazónica, Chimborazo, Ecuador, pmanobanda@uea.edu.ec
} 


\section{Resumen.}

El diseño de un proceso de producción de ácido cítrico a partir de la fermentación con Aspergillus Niger por simulación computacional, se realizó con el uso del software SuperPro Designer, los parámetros de diseño se establecieron en base a estudios previos, mismos que se ingresaron a la plataforma de cálculo, posteriormente los resultados obtenidos en el diseño se analizaron técnica y económicamente, lo que permitió determinar una cantidad de producción de ácido cítrico de 6914.69 ton/año, al usar precios FOB (Free on board) se tiene una inversión total de \$31 460000 junto a un VAN (Valor actual neto) de $\$ 165000$ y un TIR (Tasa interna de retorno) del $8.05 \%$, lo que indica que el proyecto se puede llevar a cabo y resulta rentable en un tiempo de retribución de 8 años. El proceso consta de dos etapas: fermentación y aislamiento, que deben realizarse bajo las condiciones de operación $\left(\mathrm{T}=25^{\circ} \mathrm{C}\right.$ y $\mathrm{P}=1$ atmósfera en la mayoría de las operaciones) y las condiciones óptimas de fermentación para el Aspergillus niger $\left(\mathrm{T}=35^{\circ} \mathrm{C}, \mathrm{pH}=5.8\right.$ y $\mathrm{P}=1$ atmósfera) que son adecuadas para obtener un producto de calidad que pueda ofertarse en el mercado nacional como internacional. Finalmente, se comprueba que el uso de software permite desarrollar estudios técnicos sin la necesidad de recurrir a largos períodos de tiempo con análisis experimentales en laboratorio, es decir, nos ahorra: tiempo, costos de prediseño y errores.

Palabras claves: Ingeniería Química/ Procesos Industriales/ Simulación / Fermentación/ Parámetros de operación

\section{Introducción.}

El ácido cítrico (ácido 2-hidroxi-1, 2, 3-propanotricarboxílico), es un ácido orgánico que se encuentra de forma natural en las frutas, como el limón y la naranja. Pero puede sintetizarse en laboratorio a partir de microorganismos. Se aisló por primera vez a partir del zumo de limón mezclado con cal y disolviendo el precipitado con ácido sulfúrico (Sánchez, Ortiz, \& Betancourt, 2004).

Según Kumar \& Jain (2008), en su investigación: "Solid state fermentation studies of citric acid production", alrededor del 99\% de la producción mundial de ácido cítrico se da por procesos microbianos. Se comercializa como un ácido anhidro o monohidratado, se espera una producción anual de 1.5 millones de toneladas de las cuales el $70 \%$ se utiliza en la industria de alimentos y bebidas como acidificante o antioxidante para preservar o mejorar los sabores y aromas de jugos de frutas, helados y mermeladas. $20 \%$ se usa, en la industria farmacéutica como antioxidante para conservar las vitaminas, efervescentes, correctores de $\mathrm{pH}$. El 10\% restante se utiliza en la industria química como un agente de formación de espuma para el ablandamiento y el tratamiento de los textiles. En la metalurgia, se usa para el abrillantado y eliminación del óxido en metales como el cobre y aleaciones como el latón. El ácido cítrico también se utiliza en la industria de los detergentes como un sustituto de 
fosfato, debido al menor efecto eutrófico. En Ecuador no se produce ácido cítrico a nivel industrial, este se exporta desde Alemania, Estados Unidos, China, Uruguay y otros países lo cual eleva el costo de importación, es por esto el desarrollo de un proyecto técnicoinvestigativo mediante el uso de un software que permita evaluar y diseñar un proceso de producción industrial de ácido cítrico a partir del hongo Aspergillus Niger. El elaborar una planta a escala piloto, implica costos e incluso el difícil acceso a materiales de tamaño reducido, si el proceso es complejo dificulta la determinación de los parámetros bajo los cuales se debe operar para obtener una eficiencia alta, así las evaluaciones de un proceso con el uso de simulaciones nos evitan estos gastos económicos y de tiempo con la finalidad de optimizar el proceso.

\section{Metodología}

\section{a) Ingeniera Conceptual del Proceso}

La investigación se desarrolló en forma ordenada y sistemática, iniciando con la revisión de fuentes bibliográficas específicas al tema, como libros, artículos científicos, páginas web, para definir conceptos, teorías, condiciones y variables de proceso. La simulación del proceso de producción de ácido cítrico con el uso de Aspergillus Niger, se desarrolla con el software SuperPro Designer; que es un programa computacional para realizar la simulación de procesos en estado estacionario. La simulación permite predecir la operación de un proceso cuando se han alcanzado condiciones de estacionalidad, esto facilita el estudio de la sensibilidad del sistema frente a cambios en los distintos parámetros y variables de operación (Designer, 2019).

Para iniciar la simulación de un proceso en el software, se siguen los pasos que se indican a continuación en la Figura 1:

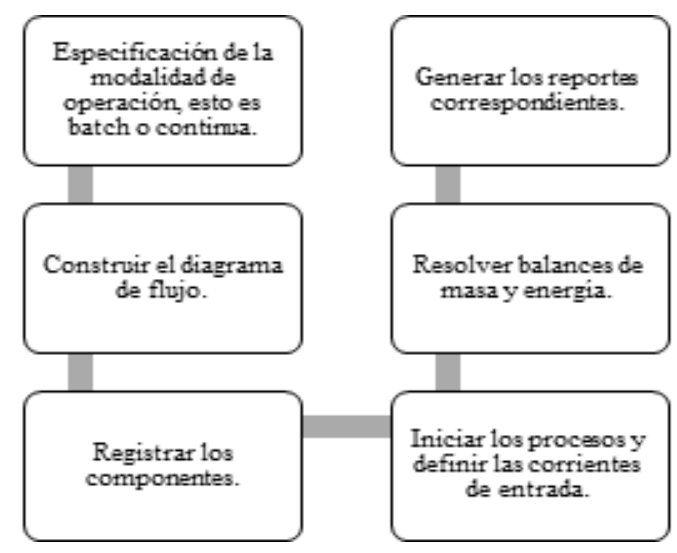

Figura 1: Modalidad de trabajo para un proceso en el simulador Realizado por: Autores, 2019

\section{b) Proceso de diseño y simulación}


El proceso de diseño y simulación de un proceso de producción sigue los pasos que se indican a continuación. En primer lugar, se inicia con la generación de ideas sobre el proceso a diseñarse, en este caso la producción de ácido cítrico por medio de fermentación, donde realiza la ingeniería conceptual del proceso, se indican las etapas, además de procesos y operaciones necesarias para cumplir con el objetivo propuesto. Una vez que se ha definido la ingeniería conceptual, se procede a realizar la planificación estratégica del proceso de diseño y posterior simulación. En segundo lugar, se desarrolla el proceso de acuerdo a la ingeniería conceptual y se evalúa las alternativas, para seleccionar la más viable. Posterior a esto, se diseñan las facilidades y se realizan los ajustes al proceso, esto en primer lugar se lo puede realizar por medio de simulación, esto nos dará como resultado la ingeniería de detalle. Finalmente, se procede a optimizar el proceso, analizar los tiempos de los ciclos y se puede programar la producción a llevarse a cabo en la planta diseñada.

\section{Diagrama General del Proceso de Producción de Ácido Cítrico}

En la Figura 2, se indica el diagrama de flujo del proceso. El diseño del proceso se realiza para obtener 6914 toneladas métricas de ácido cítrico por año en forma cristalina, que es utilizado por la industria para preservar los alimentos y bebidas, así como mejorar su sabor

\section{Producción de Ácido Cítrico}

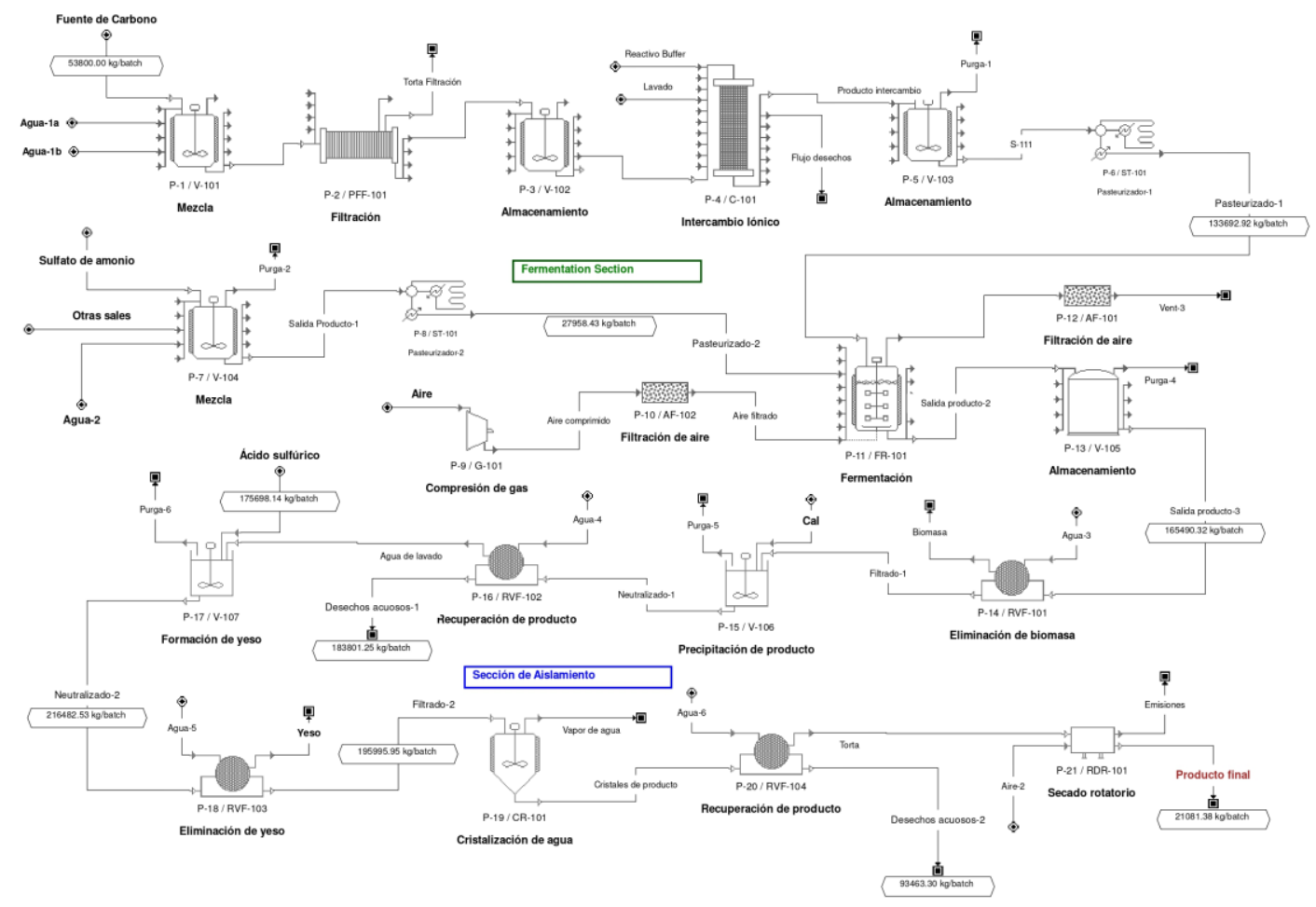

Figura 2: Diagrama del proceso de producción de ácido cítrico

Fuente: SuperPro Designer® 
La fuente de carbono que se utiliza para la fermentación es la melaza, la cual se diluye con agua hasta un 50\% de azucares fermentables (V-101). El material particulado suspendido se remueve por medio de filtración (PFF-101). Los iones metálicos, particularmente el ión de hierro, son subsecuentemente removidos por una columna cromatográfica de intercambio iónico (C-101) y la solución de materia prima purificada se esteriliza con calor en un pasteurizador (ST-101). Los nutrientes tal como zinc, fuentes de amonio, potasio, fósforo, magnesio y cobre, son disueltos en agua (V-104) y esterilizado al calor (ST-101).

Después de la esterilización, los nutrientes y la fuente de carbono se transfieren a un fermentador (FR-101). La fermentación ocurre de acuerdo a la siguiente estequiometría de masa:

$$
2\left(\mathrm{NH}_{4}\right)_{2}\left(\mathrm{SO}_{4}\right)+180 \mathrm{C}_{6} \mathrm{H}_{12} \mathrm{O}_{6}+12 \text { Nutrientes }+56 \mathrm{O}_{2} \rightarrow 16 \text { Biomasa }+30 \mathrm{CO}_{2}+154 \mathrm{C}_{6} \mathrm{H}_{8} \mathrm{O}_{7}
$$

\section{Reacción 1}

El tiempo de fermentación es de 5 días a una temperatura constante de $35^{\circ} \mathrm{C}$, con un $\mathrm{pH}$ de 5.8 hasta tener un rendimiento de reacción de 99\%. El aire necesario para mantener las condiciones aerobias de la fermentación se realiza por medio de un compresor (G-101). El agua de enfriamiento remueve el calor producido por el proceso exotérmico y mantiene la temperatura constante.

Para satisfacer la demanda de producción, la fermentación es llevada a cabo por 7 fermentadores que operan en modo alternado. Además, la planta opera todo el día y un lote de fermentación se inicia y otro se finaliza diariamente. Cada fermentador tiene un volumen de $150 \mathrm{~m}^{3}$. Una vez completada la fermentación, el caldo se descarga dentro de un tanque de retención (V-105), el cual actúa como un tanque de regulación buffer entre el lote que ingresa y la sección de salida.

Para el aislamiento de ácido cítrico, es decir, obtener este compuesto de forma pura, su purificación inicia con la remoción de la biomasa por un filtro al vacío rotatorio (RVF-101). Este líquido clarificado de la fermentación subsecuentemente fluye dentro de un reactor agitado (V-106) donde es añadido aproximadamente 1 parte de cal hidratada, $\mathrm{Ca}(\mathrm{OH})_{2}$, por 2 partes de licor esto lentamente añadida para formar el precipitado de citrato de calcio. El citrato de calcio es entonces separado por medio de un segundo filtro rotatorio al vacío (RVF102) y el filtrado libre de citrato (Desecho Acuoso-1) es enviado a un tanque de colección de desechos.

La torta de citrato de calcio se envía a otro reactor agitado (V-107), donde se añade ácido sulfúrico para formar un precipitado de sulfato de calcio (yeso). Un tercer filtro (RVF-103) remueve el precipitado de yeso y una solución de ácido cítrico impuro. Es importante cuidar el control del pH y la temperatura en la precipitación para maximizar el rendimiento de ácido 
cítrico. La solución resultante es concentrada y cristalizada en un cristalizador continuo (CR101).

\section{c) Descripción del proceso de producción de ácido cítrico}

Como se observa en la Figura 3, el proceso industrial de producción del ácido cítrico se divide en dos secciones: fermentación y aislamiento. En la primera se encuentran todos los procesos necesarios para llevar a cabo la obtención del ácido cítrico a partir de la fermentación de la melaza utilizando Aspergillus Niger. La segunda sección del proceso, corresponde a todas las operaciones necesarias, para obtener el ácido cítrico puro en forma cristalina.

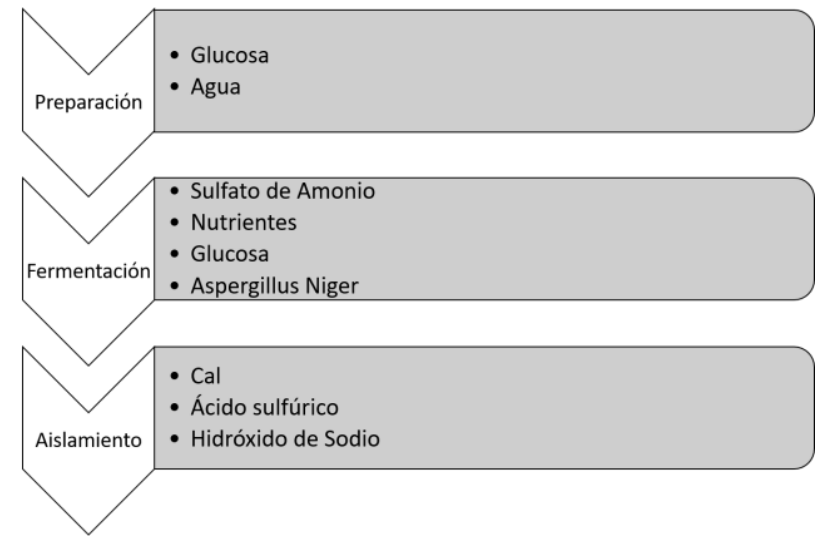

Figura 3: Secciones del proceso de producción de ácido cítrico

Realizado por: Autores, 2019

\section{Sección de Fermentación}

Para la obtención del ácido cítrico mediante fermentación con Aspergillus Niger se utiliza como materia prima la melaza, que es la fuente de carbono.

La melaza es diluida, para proceder a remover los sólidos suspendidos, además de eliminar los iones metálicos, especialmente el hierro, y finalmente esterilizarla mediante pasteurización con el fin de eliminar todos los microorganismos que pueda producir otro tipo de compuesto no deseado en la fermentación. Se añade a la melaza sustancias que mejoraran el proceso de fermentación y vuelve a ser esterilizada antes de realizarse la fermentación respectiva.

Una vez preparada la mezcla de melaza y aditivos se procede a realizar la fermentación en condiciones aeróbicas mediante inóculo de Aspergillus Niger hasta obtener el producto final y almacenarlo, para que posteriormente este producto sea dirigido hacia la sección de aislamiento. 


\section{Sección de Aislamiento}

En el producto final de la fermentación se procede a eliminar la biomasa, dando como resultado un producto totalmente líquido y sin sólidos. En este producto se elimina los sub productos y aditivos residuales del proceso de fermentación mediante precipitación, obteniéndose finalmente una corriente líquida de ácido cítrico que por procesos de cristalización y secado se consigue el producto final: ácido cítrico en forma cristalina.

\section{d) Requerimiento de materias primas}

Como materias primas se requiere: agua, melaza, sulfato de amonio, nutrientes, nitrógeno, hidróxido de sodio, hidróxido de calcio, nitrógeno, oxígeno, cal y ácido sulfúrico. Mientras que como productos se tiene: biomasa, cristales de ácido cítrico, citrato de calcio, dióxido de carbono, yeso, impurezas,

\section{e) Datos Generales del Proceso para el cálculo}

Los cálculos de diseño requieren de los datos generales del proceso de producción de ácido cítrico, estos datos son referentes a como se opera la planta de producción y el tiempo de operación de la misma como se muestra en la tabla 1.

Tabla 1: Datos generales del proceso

\begin{tabular}{lcc}
\hline Tiempo de Operación Anual & 47.90 & semana \\
\hline Tasa de Referencia de Unidad de Producción & 6914691.48 & $\mathrm{~kg} \mathrm{MP}$ \\
Tamaño del lote & 21081.38 & $\mathrm{~kg} \mathrm{MP}$ \\
Tiempo de preparación de ingredientes del lote & 199.18 & $\mathrm{~h}$ \\
Tiempo del ciclo de preparación de ingredientes & 24.00 & $\mathrm{~h}$ \\
Número de lotes por año & 328.00 & \\
\hline
\end{tabular}

*MP: Flujo total de la corriente "Producto final"

Realizado por: Autores, 2019

MP corresponde al Flujo Total de la Corriente 'Producto Final', el tiempo de operación anual, se encuentra dado en número de semanas y su cálculo se efectúa de acuerdo al tiempo de operación de la planta, cabe mencionar que, la planta opera continuamente y las operaciones son una mezcla de procesos por lote, semicontinuos o continuos.

\section{f) Requisitos de partida de materia prima por sección}


En los requisitos de materia prima se presenta la sección de producción con la respectiva materia prima de partida como es la glucosa en el caso de la fermentación y como producto activo al ácido cítrico en fase acuosa. Mientras que, la sección de aislamiento tiene como materia prima de partida el ácido cítrico producido en la sección de fermentación y como producto activo el ácido cítrico en forma de cristales.

Tabla 2: Requisitos de partida de materia prima por sección

\begin{tabular}{|c|c|c|c|c|}
\hline \multirow{4}{*}{ Sección } & \multirow{4}{*}{$\begin{array}{l}\text { Material de } \\
\text { Partida }\end{array}$} & \multirow{4}{*}{ Producto Activo } & $\begin{array}{l}\text { Rendimiento } \\
\text { Másico }\end{array}$ & Masa \\
\hline & & & $(\%)$ & Bruta \\
\hline & & & & Rendimiento \\
\hline & & & & $(\%)$ \\
\hline Sección de Fermentación & Glucosa & Ácido Cítrico & 84.53 & 84.53 \\
\hline Sección de Aislamiento & Ácido Cítrico & $\begin{array}{l}\text { Cristales de } \\
\text { Ácido Cítrico }\end{array}$ & 92.25 & 92.25 \\
\hline
\end{tabular}

Realizado por: Autores, 2019

La cantidad necesaria $(\mathrm{kg} / \mathrm{kg})$, representa el mínimo requerido para que los procesos de producción se ejecuten de forma eficiente. El rendimiento molar, rendimiento másico y rendimiento de masa bruta, corresponde al porcentaje de transformación de materia prima a producto activo mediante los procesos de producción, como se indican los valores en la tabla 2.

\section{g) Total de materias primas}

En un proceso de producción es importante conocer las cantidades que se utilizan de materia prima que interviene en el proceso de producción, para poder planificar el stock de las mismas.

Tabla 3: Total de materias primas

\begin{tabular}{lll}
\hline Material & kg/año & kg/lote \\
\hline Agua & 56701804 & 172871.354 \\
Melaza & 17646400 & 53800.000 \\
Sulfato de Amonio & 277580 & 846.281 \\
Nutrientes & 1894077 & 5774.626 \\
Nitrógeno & 49204 & 150.013 \\
NaOH (1 M) & 2617888 & 7981.366
\end{tabular}




\begin{tabular}{lll} 
Aire & 123178744 & 375544.952 \\
$\mathrm{Cal}(33 \%)$ & 13659737 & 41645.539 \\
$\mathrm{H}_{2} \mathrm{SO}_{4}(10 \% \mathrm{w} / \mathrm{w})$ & 57628992 & 175698.145 \\
TOTAL & 273654427 & 834312.276 \\
\hline
\end{tabular}

Realizado por: Autores, 2019

En la tabla 3, se presenta los kilogramos necesarios de cada materia prima por año, lote y cantidad de producto final.

\section{h) Balance general de masa del proceso de producción de ácido cítrico}

En el balance general de compuestos se presentan las sustancias químicas, impurezas y nutrientes intervienen en todo el proceso de producción.

La melaza en este balance se encuentra representada como glucosa e impurezas, también se presencia un componente NFS, el cual es Nitrógeno, Fósforo y Azufre que se utiliza para efectuar la fermentación.

\section{i) Condiciones y variables óptimas de operación}

\section{- Identificación de los equipos utilizados en el diseño}

A continuación en la tabla 4, se presentan los equipos utilizados en el proceso de producción de ácido cítrico, con sus características generales y el modo de operación, como: por lotes o batch, continuos y semicontinuos.

Tabla 4: Equipos utilizados en el diseño

\begin{tabular}{ll}
\hline Diagrama & \multicolumn{1}{c}{ Descripción } \\
\hline & $\begin{array}{l}\text { Tanque de mezcla } \\
\text { Este proceso forma parte de un sistema y permite realizar mezclas o almacena- } \\
\text { miento de líquidos que requieren ser agitados. }\end{array}$ \\
\hline & Filtro de Placa y Marco \\
& $\begin{array}{l}\text { Este proceso se utiliza en la industria química, alimentos e industrias ambientales, } \\
\text { presenta la filtración y formación de la torta por la remoción de los sólidos sus- } \\
\text { pendidos desde una lechada. }\end{array}$ \\
\hline $\begin{array}{l}\text { Cromatografía de adsorción de lecho empacado } \\
\text { Este proceso se refiere a cualquier tipo de cromatografía de adsorción, tal } \\
\text { como intercambio iónico, afinidad, HIC, fases reversas, etc., donde se encuentra } \\
\text { una columna de lecho empacado. }\end{array}$ \\
\hline
\end{tabular}




\section{Pasteurización}

Este proceso representa un pasteurizador y calcula la inactivación de microorganismos y cinética de degradación de componentes alimenticios.

\section{Fermentador por lote}

Operación que representa la secuencia de operación en un fermentador, las operaciones típicas pero no necesariamente incluyen reacciones de fermentación como estequiometria o cinética.

\section{Almacenamiento de Lote en Tanque de Fondo plano}

Este equipo permite almacenar productos líquidos, por lo que un sin número de procesos lo utilizan.

\section{Filtro Rotario al Vacío}

Este diagrama representa la filtración con

generación de torta de forma continua obteniendo altos rendimientos.

\section{Neutralización}

Este equipo representa la neutralización de un compuesto ácido o básico: $\mathrm{Pu}$ diendo especificarse cualquier número de reacciones por estequiometría.

\section{Cristalizador Continuo}

Equipo utilizado para la evaporación de una capa fina de producto, provocando la cristalización de los compuestos de interés.

\section{Secador Rotatorio}

Equipo que maneja una gran eficiencia y se utiliza en la industria química y de alimentos. El proceso de secado puede darse directamente con uso de una corriente de gas caliente o indirectamente mediante la utilización de vapor en una chaqueta.

\section{Fuente: SuperPro Designer®}

\section{- Diseño y simulación del proceso de producción de ácido cítrico}

Para el diseño y simulación se selecciona el tipo de proceso, en este caso los fermentadores tienen una operación por lotes, mientras que, el cristalizador opera de forma contínua. Luego se debe ingresar todas las sustancias que intervienen en el proceso: materia prima y productos, además de registrar las mezclas.

Posterior a esto, se realiza el diagrama del proceso junto a la secuencia de operaciones para cada equipo. Seguido se procede a revisar los parámetros de los equipos, el costo de los mismos, las características mecánicas, los aditivos, el rendimiento y si existieran comentarios.

\section{Resultados}

Para la modelación se toman como referencia datos de estudios previos para abastecer la necesidad de ácido cítrico para el Ecuador, se plantean cantidades grandes de materia prima, por tanto, se obtiene una gran producción de ácido cítrico; como se muestra en la tabla 5. 
Tabla 5: Datos modelo de simulación

\begin{tabular}{ccc}
\hline Melaza & Agua & Cristales de ácido cítrico \\
\hline $\mathrm{kg} /$ lote & $\mathrm{kg} / \mathrm{lote}$ & $\mathrm{kg} / \mathrm{lote}$ \\
53800 & 42000 & 21081.38 \\
\hline
\end{tabular}

Realizado por: Autores, 2019

En la tabla 5, se muestran los principales valores que se obtienen en la simulación del modelo para la producción de ácido cítrico. Se muestran cantidades de materia prima, producción total de ácido cítrico, total de residuos y el resumen de costos para el análisis económico.

Tabla 6: Datos técnicos y económicos de los cuatro modelos simulados

\begin{tabular}{ll}
\hline & \multicolumn{1}{c}{ Datos } \\
\hline Melaza (kg/año) & 17646400 \\
Agua (kg/año) & 56701804 \\
Total de materias primas (kg/año) & 273654427 \\
Tasa de producción Ac. Cítrico (kg/año) & 6914691.48 \\
Tasa de producción de Ac. Cítrico (ton/año) & 6914 \\
Costos FOB de equipos (\$) & 7018000 \\
Inversión total de capital (\$) & 31460000 \\
Margen bruto (\%) & 16.09 \\
Retorno sobre la inversión (\%) & 11.28 \\
Tiempo de retribución (años) & 8.86 \\
TIR (\%) & 8.05 \\
VAN (8\%) & 165000 \\
\hline
\end{tabular}

Realizado por: Autores, 2019

En base a la Tabla 6, se define que el modelo de simulación es óptimo para la producción de ácido cítrico por vía de fermentación con el uso de Aspergillus niger, este modelo es viable desde el punto de vista técnico y económico, se tiene una producción acorde a las necesidades del mercado, la inversión es menor y la ganancia es en un tiempo aproximado de 8 años. 
- Estimación de costos de las operaciones del proyecto

\section{Base de costos.}

Los resultados de los costos presentados en esta sección se encuentran en base a costos del año 2012, en función al año de los costos encontrados en bibliografía y proveedores de equipos. Además, los costos se encuentran expresados en dólares y en precios FOB (Free On Board).

\section{Resumen de costos}

En este apartado se presenta el resumen de costos de toda la planta de producción de ácido cítrico, para lo cual cada ítem tiene un valor y se encuentra expresado en sus unidades respectivamente.

Tabla 7: Resumen de costos

\begin{tabular}{|c|c|c|}
\hline Inversión Total de Capital & 31460000 & $\$$ \\
\hline Costo de Operación & 31460000 & \$/año \\
\hline Costo neto de Operación & 11605000 & \$/año \\
\hline Ingresos & 13829000 & \$/año \\
\hline Tasa de Costo Base Anual & 6914691 & $\mathrm{~kg}$ MP/año \\
\hline Costo unitario de Producción & 1.68 & $\$ / \mathrm{kg} \mathrm{MP}$ \\
\hline Costo neto de Producción Unitaria & 1.68 & $\$ / \mathrm{kg} \mathrm{MP}$ \\
\hline Ingresos de Producción Unitaria & 2.00 & $\$ / \mathrm{kg} \mathrm{MP}$ \\
\hline Margen Bruto & 16.09 & $\%$ \\
\hline Retorno sobre la inversión & 11.28 & $\%$ \\
\hline Tiempo de retribución & 8.86 & Años \\
\hline TIR (Después de Impuestos) & 8.05 & $\%$ \\
\hline VAN (con $8.0 \%$ Intereses) & 165000 & $\$$ \\
\hline
\end{tabular}

Realizado por: Autores, 2019

En la Tabla 7, el flujo total de la corriente de producto final está representado por MP, mientras que el TIR corresponde a la Tasa Interna de Retorno que se refiere a la rentabilidad que genera un proyecto determinado cuando sus flujos de caja son periódicos. El VAN es el valor actual neto, que es el cálculo a valor presente, el dinero que una inversión generará en el futuro.

\section{Discusión de resultados}


Se realizó un modelo de simulación para la producción de ácido cítrico por fermentación con el uso de Aspergillus Níger, en el que se ingresan cantidades de materia prima, tamaño de equipos, lo que permite obtener los costos de producción y la inversión total de la planta.

El diseño del proceso de producción consta de dos secciones, la sección de fermentación en donde se utiliza como materia prima la melaza como fuente de carbono (53 $800 \mathrm{~kg} / \mathrm{lote}$ ) para esta etapa. A continuación en un tanque de mezcla se diluye la melaza (42000 kg/lote), con el fin de remover los sólidos suspendidos (Filtro) y eliminar los iones metálicos (columna de intercambio iónico), es necesario pasteurizar con el fin de matar los microorganismos no deseados y evitar la formación de otros compuestos. Al fermentador ingresa también una mezcla de sulfato de amonio y nutrientes (nitrógeno, fósforo y azufre), con el fin de mejorar el producto para la siguiente sección. Con esta mezcla que se prepara y los aditivos se realiza la fermentación en condiciones aeróbicas mediante inóculo de Aspergillus Niger, bajo un pH de 5.8 y una temperatura de $35^{\circ} \mathrm{C}$ durante cinco días, hasta obtener el producto final, almacenarlo y dirigirlo hacia la siguiente sección: la de asilamiento.

En la sección de aislamiento se elimina la biomasa, subproductos y aditivos residuales (Filtro al vacío rotatorio), obteniendo un producto líquido libre de sólidos (ácido cítrico), que por cristalización y secado se obtiene el ácido cítrico en forma cristalina (21 $081.38 \mathrm{~kg} / \mathrm{lote})$.

Según Rivada (2008), en su estudio planta industrial de producción de ácido cítrico a partir de melazas de remolacha la mejor cepa que permite el crecimiento de Aspergillus Niger es la ATTC 11414, en un pH ácido de 5.8 y una temperatura de $30 \circ \mathrm{C}$, en la investigación los fermentadores operan a $35^{\circ} \mathrm{C}$ durante cinco días con el fin de producir la cantidad ideal de ácido cítrico.

El simulador SuperPro Designer según Cara, Gómez\&Martínez en su estudio “Aplicación de SuperPro Designer en el análisis de sensibilidad en instalaciones para la producción de biocombustibles y bioproductos", menciona que ésta plataforma presenta una herramienta de análisis de costes y emisiones lo que la hace muy versátil para su utilización en diferentes asignaturas relacionadas con los procesos fermentativos, ambientales, energías renovables y de producción química; este estudio evidencia la gran utilidad que proporciona el simulador al permitir diseñar el proceso de producción de ácido cítrico por fermentación con el uso del hongo Aspergillus Niger.

La inversión total de la planta para el modelo es de \$ 31460 000, el Van (Valor neto actual) que es de $\$ 165$ 000, el TIR (Tasa interna de retorno) de 8.05\% en un tiempo de retribución del capital de 8 años. Según Mete (2014), en su estudio "Valor actual neto y tasa de retorno: su utilidad como herramientas para el análisis y evaluación de proyectos de inversión", manifiesta que el VAN y el TIR son imprescindibles para garantizar un correcto análisis financiero con el fin de asegurar la estabilidad y proyección futura de las organizaciones, en 
este caso los valores del VAN y TIR al ser positivos, indican que la inversión en el proyecto es rentable, debido a que se recupera el capital y existe ganancia en un tiempo estimado.

Al no existir estudios previos similares a esta investigación basada en una simulación, no se establecen comparaciones.

El impacto ambiental que produce el proceso no representa un daño considerable para el medio ambiente, se tiene $141959226 \mathrm{~kg} / \mathrm{año}$ de residuos líquidos, orgánicos, acuosos y emisiones; los residuos provenientes de aguas de lavado son los que se encuentran en mayor cantidad (103 $190859 \mathrm{~kg} / \mathrm{año}$ ), por esta razón se debe tratar los residuos previo a su descarga o buscar una alternativa para usarlos y de esta manera disminuir la contaminación leve que provocan, el costo total para el tratamiento de residuos es de $\$ 629648$, que se incluyen en la inversión total.

\section{Conclusiones.}

- El diseño del proceso de producción de ácido cítrico por fermentación con el uso de Aspergillus Níger consta de dos secciones: fermentación y aislamiento. La sección de fermentación cuenta con trece operaciones entre filtración, mezcla, intercambio iónico y fermentación en sí; La sección de asilamiento tiene diez operaciones entre filtración al vacío, mezcla, cristalización y secado. La cepa de Aspergillus Níger que más se utiliza para la producción de ácido cítrico a nivel industrial es la ATTC 11414.

- La simulación del proceso de producción de ácido cítrico indica que es viable, acorde al análisis económico; se asemeja más a un proyecto real que a nivel técnico y económico se podría llevar a cabo.

- Para que el proceso de producción de ácido cítrico se realice de manera adecuada se debe controlar las condiciones y variables de operación; para la sección de fermentación, las variables a tener en cuenta son: temperatura constante para la mayoría de operaciones de $25^{\circ} \mathrm{C}$ y a 1 atmósfera de presión. La fermentación del hongo (Aspergillus Niger), se da a un $\mathrm{pH}$ de 5.8; a una temperatura óptima de $35^{\circ} \mathrm{C}$, durante cinco días; se recomienda usar 7 fermentadores para evitar cuellos de botella y lograr que el proceso sea continuo. Para la sección de aislamiento, la temperatura opera entre un rango más elevado respecto al anterior $\left(25-110^{\circ} \mathrm{C}\right)$, y la presión es de 1 atmósfera.

- La inversión total de capital para el modelo óptimo es de \$31460 000; los índices de rentabilidad que se determinan son: la tasa interna de retorno (TIR) que refleja un valor de $8.05 \%$, el Valor Actual Neto es de $\$ 165$ 000; en un tiempo de retribución de 8 años, estos valores indican que el proyecto es rentable y es factible invertir.

\section{Referencias bibliográficas.}

Baker, S. (2006). Aspergillus niger genomics: Past, present and into the future. Medical Mycology , 17-21.

Blanch, H. W., \& Clark, D. S. (1997). Biochemical Engineering. New York: Dekker. 
Cara, J., Gómez, X., Martínez, E., Rosas, J., \& Fernández, C. (s/f.). Aplicación de SuperPro Designer en el análisis de sensibilidad en instalaciones para la producción de biocombustibles y bioproductos. Universidad de León, Departamento de Química y Fisica Aplicadas, León. Obtenido de http://www.coddiq.es/cidiq2016/wp-content/uploads/2015/02/P2.pdf

Costa, J., Cercera, S., F., C., Esplugas, S., Mans, C., \& Mata, J. (1991). Curso de Ingeniería Química. Barcelona: Editorial Reverté.

Coulson, J., \& Richardson, J. (1982). Ingeniería Química. Barcelona: Editorial Reverté.

Crueger, W., \& Crueger, A. (1989). Biotechnology-A Textbook of Industrial Microbiology, 2nd ed. Sunderland, Massachusetts: Sinauer Associates, Inc.

Drake, J. (2008). Proceso de desarrollo de aplicaciones de software. Santander.

García, L. (2013). Distribución T-student. Perú: Universidad Nacional Federico Villareal.

García, M., Quintero, R., \& López, A. (2004). Biotecnología Alimentaria. México D.F.: Limusa.

Green Planet Technologies. (2019). Green Planet Technologies. Obtenido de Green Planet Technologies

Baker, S. (2006). Aspergillus niger genomics: Past, present and into the future. Medical Mycology, 17-21.

Blanch, H. W., \& Clark, D. S. (1997). Biochemical Engineering. New York: Dekker.

Cara, J., Gómez, X., Martínez, E., Rosas, J., \& Fernández, C. (s/f.). Aplicación de SuperPro Designer en el análisis de sensibilidad en instalaciones para la producción de biocombustibles y bioproductos. Universidad de León, Departamento de Química y Fisica Aplicadas, León. Obtenido de http://www.coddiq.es/cidiq2016/wpcontent/uploads/2015/02/P2.pdf

Costa, J., Cercera, S., F., C., Esplugas, S., Mans, C., \& Mata, J. (1991). Curso de Ingeniería Química. Barcelona: Editorial Reverté.

Coulson, J., \& Richardson, J. (1982). Ingeniería Química. Barcelona: Editorial Reverté.

Crueger, W., \& Crueger, A. (1989). Biotechnology-A Textbook of Industrial Micro-biology, 2nd ed. Sunderland, Massachusetts: Sinauer Associates, Inc.

Drake, J. (2008). Proceso de desarrollo de aplicaciones de software. Santander.

García, L. (2013). Distribución T-student. Perú: Universidad Nacional Federico Villareal. 
García, M., Quintero, R., \& López, A. (2004). Biotecnología Alimentaria. México D.F.: Limusa.

Green Planet Technologies. (2019). Green Planet Technologies. Obtenido de Green Planet Technologies

Hernández, A. (2003). Microbiología Industrial. San José: Universidad Estatal a Distancia.

Himmeblau, D. (2002). Principios Básicos y Cálculos de Ingeniería Química. México: Pearson Educación.

HR Engineering Co. (2019). HR Engineering Co., India. Obtenido de https://www.indiamart.com/hr-engineering-corporation/

Jagani. (2010). An Overview of Fermenter and the Design Considerations to Enhance Its Productivity. Pharmacologyonline, 261-301.

Jiménez, A. (2003). Diseño de Procesos en Ingeniería Quimica. Barcelona: Editorial Reverté.

Kumar, A., \& Jain, V. (2008). Solid state fermentation studies of citric acid production . African Journal of Biotechnology, 644- 650.

Martínez, V. (2003). Simulación de procesos en Ingeniería Química. México: Plaza y Valdes.

McCabe, W., \& Smith, J. (2003). Operaciones básicas de Ingeniería Química. Sevilla: Editorial Reverté.

Mujundar, A. (2016). Handbook of Industrial Driying. Boca Raton, FL: Taylor \& Francis Group.

Mullin, J. (1972). Crystallitation. London: Butterworth \& Co.

Muñoz, A., Sáenz, A., López, L., Cantú, L., \& Barajas, L. (2014). Acido cítrico: Compuesto interesante. Revista Científica de la Universidad Autónoma de Coahuila.

Okewale, A., Igbokwe, P., \& Babayemi, K. (2015). Design of Pilot Plant Packed Column for the Dehydration of Water from Ethanol-Water Mixtures. Advances in Chemical Engineering and Science, 152-157.

Palacio, L., Tapias, H., \& Saldarriaga, C. (2005). Métodos y algoritmos de diseño en ingeniería química. Antioquia: Universidad de Antioquia.

Papagianni, M. (2007). Advances in citric acid fermentation by Aspergillus niger: Biochemical aspects, membrane transport and modeling. Biotecnology Advances, 244-263. 
Pérez, O., Ley, N., Regla , K., \& González, E. (2016). Oportunidades de producción de ácido cítrico por vía fermentativa a partir de sustratos azucarados en Cuba. Centro Azúcar, 85-99.

Press Release Distribution. (2017). PRLOG. Obtenido de http://www.prlog.org/11293569china-citric-acid-price-trend-outlook-2011.html

Rivada, F. (2008). Planta industrial de producción de ácido cítrico a partir de melazas de remolacha. Universidad de Cádiz, Facultad de Ciencias.

Roberts, L. (1979). Encyclopedia of Chemical Processing and Design (Vol. 8). New York: Dekker.

Sáez, A., Flórez, L., \& Cadávid, A. (2002). Caracterización de una cepa nativa de Aspergillus Niger y Evaluación de la producción de ácido cítrico. Revista Universidad EAFIT.

Sánchez, O., Ortiz, M., \& Betancourt, A. (2004). Obtención de ácido cítrico a partir de suero de leche por fementación con Aspergillus spp. Revista Colombiana de Biotecnología, 43-54.

Schuster, E., Dunn, N., \& Frisvad, J. (2002). On the safety of Aspergillus Niger- a review. Appl Microbiol Biotechnol, 428-435.

Vázquez, R., Miranda, M., \& Muñoz, G. (2014). Balance de materia y energía de Procesos Industriales. México: Grupo Editorial Patria.

Weissermel, K., \& Hans-Jurgen, A. (1981). Química Orgánica Industrial. Barcelona: Editorial Reverté. 


\section{PARA CITAR EL ARTÍCULO INDEXADO.}

Guadalupe Alcoser, M. A., Parada Rivera, M. M., Flores Fiallos, L. M., \& Manobanda Pinto, P. M. (2020). Diseño de un proceso de producción de ácido cítrico por simulación computacional. Ciencia Digital, 4(1), 54-71. https://doi.org/10.33262/cienciadigital.v4i1.1063

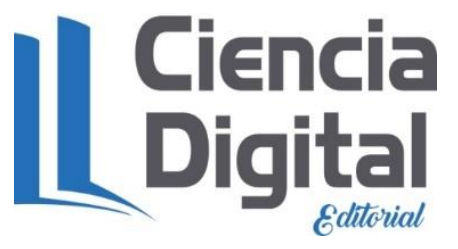

El artículo que se publica es de exclusiva responsabilidad de los autores y no necesariamente reflejan el pensamiento de la Revista Ciencia Digital.

El artículo queda en propiedad de la revista y, por tanto, su publicación parcial y/o total en otro medio tiene que ser autorizado por el director de la Revista Ciencia Digital.
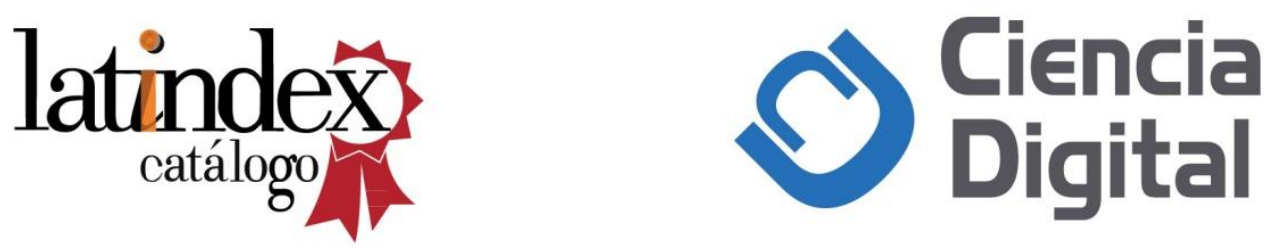